\title{
Millimetre Astronomy from the High Antarctic Plateau: Site Testing at Dome C
}

\author{
L. Valenziano ${ }^{1}$ and G. Dall'Oglio ${ }^{2}$ \\ ${ }^{1}$ CNR-TeSRE, via P. Gobetti 101, Bologna, I-40129 Italy \\ valenziano@tesre.bo.cnr.it \\ ${ }^{2}$ Dipartimento di Fisica, Università di Roma TRE, \\ via della Vasca Navale 84, Roma, I-00154 Italy \\ dalloglio@amaldi.fis.uniroma3.it \\ Received 1999 January 14, accepted 1999 April 21
}

\begin{abstract}
Preliminary site testing results at Dome C (Antarctica) are presented, using both Automatic Weather Station (AWS) meteorological data (1986-1993) and Precipitable Water Vapour (PWV) measurements made by the authors. A comparison with the South Pole and other sites is made. The South Pole is a well established astrophysical observing site, where extremely good conditions are reported for a large fraction of time during the year. Dome C, where Italy and France are building a new scientific station, is a potential observing site in the millimetre and submillimetre range. AWS are operating at both sites and they have been continuously monitoring temperature, pressure and wind speed and direction for more than ten years. Site testing instruments are already operating at the South Pole (AASTO, Automated Astrophysical Site-Testing Observatory), while light experiments have been running at Dome C (APACHE, Antarctic Plateau Anisotropy CHasing Experiment) during summertime. A direct comparison between the two sites is planned in the near future, using the AASTO. The present analysis shows that the average wind speed is lower at Dome $\mathrm{C}\left(\sim 1 \mathrm{~m} \mathrm{~s}^{-1}\right)$ than at the South Pole $\left(\sim 2 \mathrm{~m} \mathrm{~s}^{-1}\right)$, while temperature and PWV are comparable.
\end{abstract}

Keywords: site testing — methods: observational and data analysis

\section{Introduction}

The high Antarctic Plateau is considered the best site on Earth for astrophysical observations in the millimetre and submillimetre wavelength ranges (Burton et al. 1994). In the last few years many astrophysical experiments have been deployed on this continent (Ruhl et al. 1995; Viper Home Page; Balm 1996; Storey, Ashley \& Burton 1996; Valenziano et al. 1998) and large telescope projects are presently being developed (Stark 1998). The main astrophysical observing site, already operational, is the US Amundsen-Scott station at the South Pole (SP hereafter), where CARA (Center for Astrophysical Research in Antarctica) has installed a large laboratory. Data on the observing conditions at SP have already been reported in the literature (Dragovan et al. 1990; Chamberlin \& Bally 1994; Chamberlin 1995; Chamberlin, Lane \& Stark 1997; Lane 1998), while sophisticated tests are being performed by Australian researchers, using an automated instrument (Storey, Ashley \& Burton 1996).

Italy has been deeply involved in astrophysical activities in Antarctica since 1987. The Italian
Antarctic Program (PNRA, Programma Nazionale di Ricerche in Antartide) has established an astrophysical observatory (OASI, Osservatorio Antartico Submillimetrico e Infrarosso, Dall'Oglio et al. 1992) at the Italian station Baia Terra Nova $\left(74^{\circ} 41^{\prime} 36^{\prime \prime} \mathrm{S}\right.$, $\left.164^{\circ} 05^{\prime} 58^{\prime \prime} \mathrm{E}\right)$. A $2 \cdot 6 \mathrm{~m}$ submillimetre telescope is operating there during the southern summer season. Many facilities are available, including nitrogen and helium liquefiers, mechanical workshops, electronic and cryogenic laboratories. The site quality is comparable to that of mid-latitude mountain observatories for millimetre (Dall'Oglio et al. 1988) and mid-infrared observations (Valenziano 1996).

In 1994, Italy and France started a program to build a permanent scientific station (Concordia) on the high Antarctic Plateau, at Dome C, hereafter $\mathrm{DC}\left(75^{\circ} 06^{\prime} 25^{\prime \prime} \mathrm{S}, 123^{\circ} 20^{\prime} 44^{\prime \prime} \mathrm{E}\right)$. Domes are regions more elevated than the rest of the continent (DC is at $3280 \mathrm{~m}$ ), barring the Trans-Antarctic Mountains. The highest is Dome A (4100 m), potentially the best observing site on the planet, but it is very difficult to reach.

In December 1995, a test experiment was run to directly compare the short-term atmospheric stability (the so-called sky noise) between DC and the Italian 
station on the coast. Raw data show reductions in rms noise of factors of 3 and 10 at $\lambda=2 \mathrm{~mm}$ and $\lambda=$ $1.2 \mathrm{~mm}$ respectively (Dall'Oglio 1997). In December 1996 the APACHE96 (Antarctic Plateau Anisotropy CHasing Experiment; Valenziano et al. 1997, 1998) was set up at DC. Preliminary data analysis shows good atmospheric stability in terms of sky noise at mm wavelengths. The atmospheric PWV content was measured during the latter mission.

The AASTO experiment, presently running at SP, is planned to be moved to DC in the next few years. It will allow a careful assessment of the observational quality at DC over a wide wavelength range. However, some interesting information can be obtained from meteorological data, taking advantage of the good statistics based on data collected over eight years.

The AWS project, started in the early 1980s, installs automatic units in remote areas of the Antarctic continent. The main objective of this program is to support meteorological research by unattended, low-cost, data collecting stations. AWS units operate continuously throughout the year. AWS data for all the stations are publicly available at the University of Wisconsin, Madison. One AWS unit is located a few kilometres from the Concordia Station site. It therefore shares the same atmospheric conditions as the planned astrophysics observatory. Another unit is at the geographical SP (Clean Air, elevation $2836 \mathrm{~m}$ ). Therefore it is possible to compare the two sites on the basis of homogeneous data.

The main goal of this paper is to present data on DC conditions and to compare them with those of well established observing sites. These data are useful for planning experiments and for stimulating a deeper exploration of this interesting and promising observing site.

\section{Automatic Weather Stations}

AWS were developed by the Radio Science Laboratory at Stanford University. The basic AWS units measure air temperature, wind speed and wind direction at a nominal height of $3 \mathrm{~m}$ above the surface, and air pressure at the electronic enclosure about 1.75 $\mathrm{m}$ above the surface. The height is only nominal, due to possible snow accumulation. Some AWS units can also measure humidity and vertical air temperature difference, but these sensors are not available at either the DC or SP station. Data are transmitted to a NOAA (National Oceanic and Atmospheric Administration) satellite and then stored at the University of Wisconsin (Keller et al. 1997). More details on the AWS program are available at http://uwamrc.ssec.wisc.edu/aws/.

\section{AWS Data Analysis}

AWS data used in this work are already binned in three-hour intervals. This is useful in order to evaluate the stability of the observing conditions over a reasonably short interval. Some data can be missing, due to instrumental or transmission failures. Data were further averaged over one-month intervals. The typical uncertainty in the monthly averages is $15 \%$ (standard deviation) for temperature, 1-6\% for pressure and 50-75\% for wind speed. Plots of these data are shown in Figures 1, 3 and 5. Statistical distributions of the whole data set have also been calculated. Data have been binned intervals of $1^{\circ}$ for temperature, $1 \mathrm{hPa}$ for pressure, $1 \mathrm{~m} \mathrm{~s}^{-1}$ for wind speed and $10^{\circ}$ for wind azimuth. Histograms for temperature, pressure, wind azimuth and wind speed are presented in Figures 2, 4, 6 and 7. Monthly plots of the whole data set and data distributions, along with a table with median values and mean absolute deviation, are reported elsewhere (Valenziano 1997).

\section{Precipitable Water Vapour Measurements}

To the best of our knowledge, the first published measurement of the DC PWV content of the atmosphere was performed by the authors in January 1997 (Valenziano et al. 1998). The instrument used was a portable photometer (Volz 1974), with an accuracy of $20 \%$. The limited sensitivity of the instrument allowed only upper limits to be set in some cases. Data are presented in Figure 8. The average PWV at DC is around $0.6 \mathrm{~mm}$.

A comparison between DC, SP, Atacama and Mauna Kea sites is presented in Table 1. Data for DC were measured by the authors (in one month), while quartile data for the other site are from Lane (1998). Results for the Vostok station (elevation $3488 \mathrm{~m}$; Townes \& Melnick 1990) in summer do not differ significantly from those reported here, but values of less than $0.1 \mathrm{~mm}$ were measured during winter. We have also calculated the $225 \mathrm{GHz}$ and $492 \mathrm{GHz}$ opacities from the 50th percentile PWV data, using a model derived from the SP data (Lane 1998). We used the following relations:

$$
\begin{aligned}
& \tau_{0}(225 \mathrm{GHz})=0 \cdot 030+0 \cdot 069 \mathrm{PWV}(\mathrm{mm}), \\
& \tau_{0}(492 \mathrm{GHz})=0 \cdot 33+1 \cdot 49 \mathrm{PWV}(\mathrm{mm})
\end{aligned}
$$

and we evaluated the corresponding transmissions at the zenith as $T=\exp \left(-\tau_{0}\right)$. These values are reported in Table 1, along with available data for other sites (from Lane 1998).

\section{Discussion}

The analysis of the AWS data set and the PWV measurements shows the following main results:

- DC and SP average temperatures are comparable, ranging between typical values of $-65^{\circ} \mathrm{C}$ in winter and $-26^{\circ} \mathrm{C}$ in summer. The median value for 
Table 1. Quartile values of PWV and 225 and $492 \mathrm{GHz}$ opacity and transmissions at DC, SP, Mauna Kea and Atacama Data for sites other than DC are from Lane (1998). Values in the first line for DC are calculated for measurements only, while those in the second line include upper limits. Values for the zenith opacity, referred to $50 \%$ quartile, for SP, Mauna Kea and Atacama are measured with sky-dips. DC data are calculated from $50 \%$ quartile PWV

\begin{tabular}{|c|c|c|c|c|c|c|c|}
\hline \multirow[t]{2}{*}{ Site } & \multicolumn{3}{|c|}{ PWV (mm) } & \multirow{2}{*}{$\begin{array}{c}\tau_{0} \\
(225 \mathrm{GHz})\end{array}$} & \multirow{2}{*}{$\begin{array}{c}\text { Transm. } \\
(225 \mathrm{GHz})\end{array}$} & \multirow{2}{*}{$\begin{array}{c}\tau_{0} \\
(492 \mathrm{GHz})\end{array}$} & \multirow{2}{*}{$\begin{array}{c}\text { Transm. } \\
(492 \mathrm{GHz})\end{array}$} \\
\hline & $25 \%$ & $50 \%$ & $75 \%$ & & & & \\
\hline Dome C (excluding UL) & $0 \cdot 38$ & $0 \cdot 52$ & $0 \cdot 68$ & $0 \cdot 066^{1}$ & $0 \cdot 94$ & $1 \cdot 10^{1}$ & $0 \cdot 33$ \\
\hline Dome C (including UL) & $0 \cdot 47$ & $0 \cdot 64$ & $0 \cdot 78$ & $0 \cdot 074^{1}$ & $0 \cdot 93$ & $1 \cdot 28^{1}$ & $0 \cdot 28$ \\
\hline South Pole (winter) & $0 \cdot 19$ & $0 \cdot 25$ & $0 \cdot 32$ & $0 \cdot 046$ & $0 \cdot 95$ & $0 \cdot 70$ & $0 \cdot 50$ \\
\hline South Pole (summer) & $0 \cdot 34$ & $0 \cdot 47$ & $0 \cdot 67$ & $0 \cdot 062$ & $0 \cdot 94$ & $1 \cdot 03$ & $0 \cdot 36$ \\
\hline Mauna Kea (winter) & $1 \cdot 05$ & $1 \cdot 65$ & $3 \cdot 15$ & $0 \cdot 076$ & $0 \cdot 93$ & $1 \cdot 32$ & $0 \cdot 28$ \\
\hline Mauna Kea (summer) & $1 \cdot 73$ & $2 \cdot 98$ & $5 \cdot 88$ & $0 \cdot 129$ & $0 \cdot 88$ & $2 \cdot 38$ & $0 \cdot 09$ \\
\hline Atacama (winter) & $0 \cdot 68^{2}$ & $1 \cdot 00^{2}$ & $1 \cdot 60^{2}$ & $0 \cdot 044$ & $0 \cdot 96$ & & \\
\hline Atacama (summer) & $1 \cdot 10^{2}$ & $2 \cdot 00^{2}$ & $3 \cdot 70^{2}$ & $0 \cdot 077$ & $0 \cdot 93$ & & \\
\hline
\end{tabular}

${ }^{1}$ From PWV values, using a model (see text). ${ }^{2} \mathrm{PWV}$ values are calculated from the $225 \mathrm{GHz}$ opacity (Lane 1998 ).

Table 2. Comparison between AWS data for two Antarctic sites, Mauna Kea (Hawaii Islands) and Atacama desert (Chile)

\begin{tabular}{|c|c|c|c|c|}
\hline & Dome C & South Pole & Mauna Kea & Atacama \\
\hline Elevation (m) & 3280 & 2836 & 3750 & 5000 \\
\hline Average pressure (hPa) & 644 & 682 & 650 & 550 \\
\hline Median wind speed $\left(\mathrm{ms}^{-1}\right)$ & $2 \cdot 1$ & $3 \cdot 6$ & $4 \cdot 5$ & $6 \cdot 0$ \\
\hline Maximum wind speed $\left(\mathrm{ms}^{-1}\right)$ & $15 \cdot 9$ & $18 \cdot 5$ & $28 \cdot 8$ & $33 \cdot 0$ \\
\hline
\end{tabular}

both sites is $-53^{\circ} \mathrm{C}$, while the correlation of monthly average temperatures between DC and $\mathrm{SP}$ is $96 \%$.

- The pressure is always lower at DC than at SP. Median values are $644 \mathrm{hPa}$ and $682 \mathrm{hPa}$ respectively. Monthly averaged pressure data show a correlation of $92 \%$ between the two sites.

- The wind speed is very low at both sites, with a maximum speed of $15.9 \mathrm{~m} \mathrm{~s}^{-1}$ at DC and 18.9 $\mathrm{ms}^{-1}$ at SP.

- A Kolmogorov-Smirnov test shows that wind speed distributions for the two sites are different. The correlation between monthly averaged wind speed data is less than $30 \%$. The 50th percentile values for wind speed, evaluated for the whole data set, are $1 \mathrm{~ms}^{-1}$ at DC and $2 \mathrm{~ms}^{-1}$ at SP.

- Wind azimuth distributions are different: the prevalent direction is approximately azimuth 180 at DC and between azimuth 0 and 90 at SP.

- PWV values measured at DC in January 1997 are comparable with SP values in the same season and lower than those measured at other sites.

In Table 1 and Table 2 our results for Antarctica (over eight years) are compared with data from a well established observing site, Mauna Kea (Hawaii Islands), and a future important site in the Atacama desert (Northern Chile). Data for these latter sites are taken from Holdaway (1996).

Some conclusions can be derived from these results:

- Lower wind regimes at the Antarctic sites result in lower turbulence, implying a smaller contamination on observations. It is worth considering that most of the sky-noise at infrared and millimetre wavelengths is induced by convective motion and wind-driven turbulence in the lowest layers of the atmosphere, where the bulk of water vapour is found (Smoot et al. 1987; Ade et al. 1984; Andreani et al. 1990). While the former needs further investigation (Argentini 1998; Burton 1995), the latter is minimal on the Antarctic Plateau.

- Lower average wind speed reduces pointing errors for large antennas (see Holdaway 1996).

- In terms of wind speed, conditions at DC are better than those at SP.

- The high Antarctic Plateau is the driest observing site on Earth.

- DC and SP pressure and temperature conditions are strongly correlated, indicating that they share similar meteorological conditions. It is possible to infer that $\mathrm{PWV}$ values at $\mathrm{DC}$ during wintertime are similar to SP ones in the same season.

- $225 \mathrm{GHz}$ and $492 \mathrm{GHz}$ opacities for DC, calculated using a model valid for SP, show results similar to those for SP and better than those at Mauna Kea, when comparable water vapour amounts are considered.

\section{Conclusions}

An analysis of available site quality data for DC has been accomplished, including PWV measurements made by the authors. A comparison with SP and other sites has been performed.

AWS data show that the quality of DC and SP is comparable, at least for meteorological conditions. DC seems to be a more suitable site in terms of wind speed (which is related to atmospheric turbulence). 
Dome C

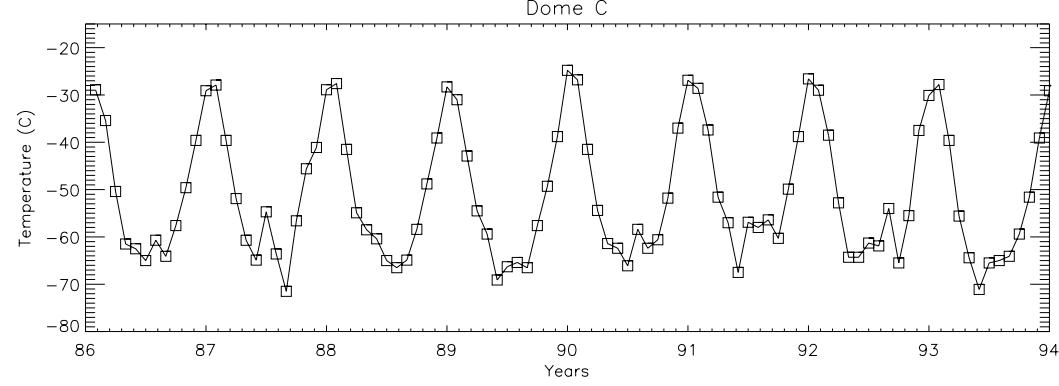

South Pole

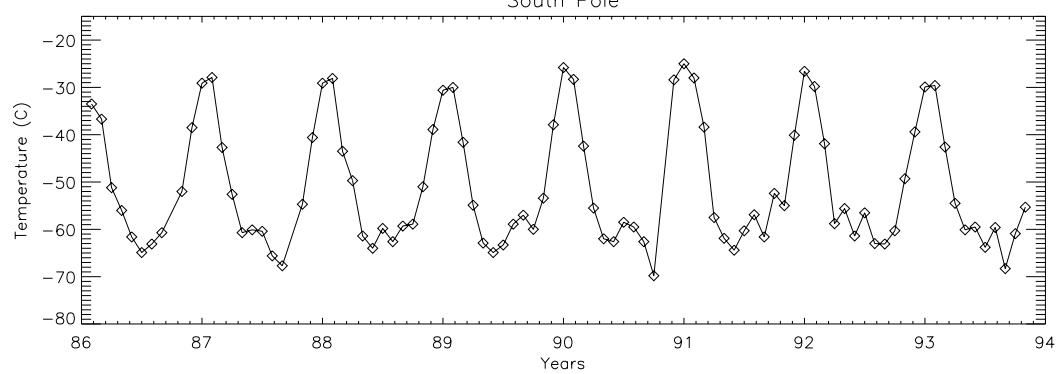

Figure 1-Monthly averaged temperature at DC (upper panel) and SP (lower panel). The correlation between the two data sets is $96 \%$.

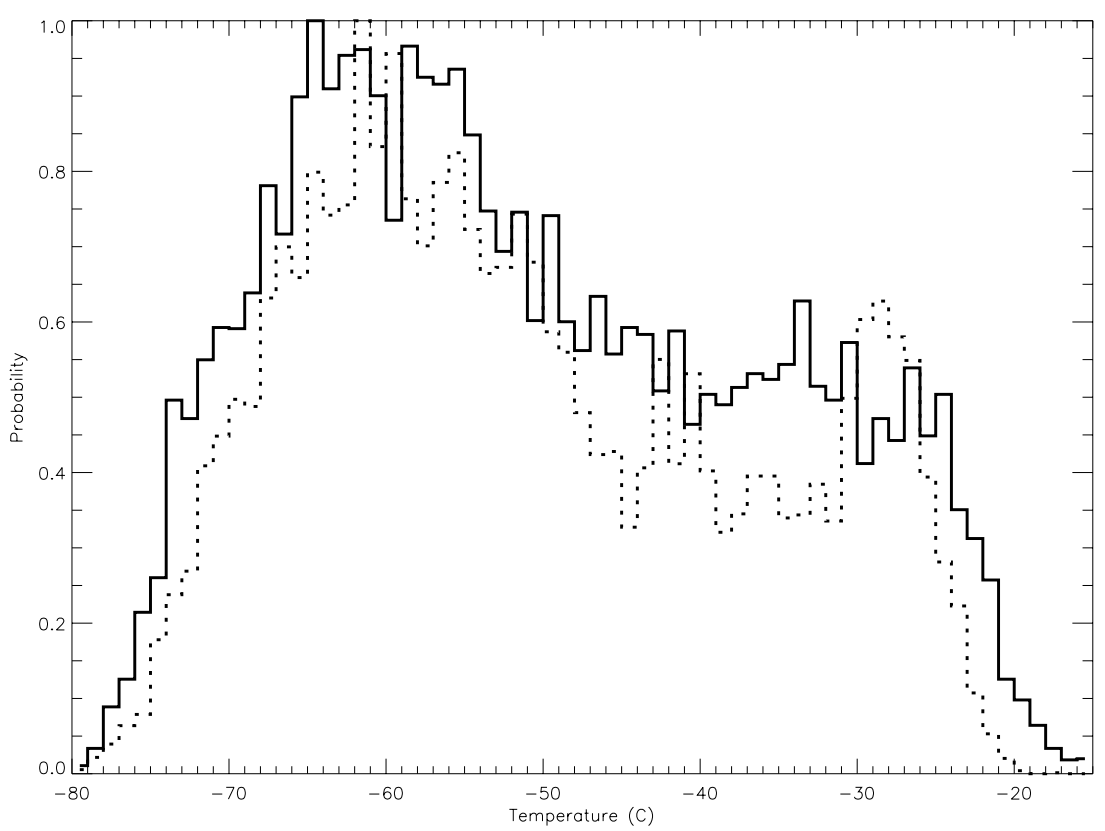

Figure 2-Temperature distributions for DC (continuous line) and SP (dotted line). The two distributions are consistent within errors. Median temperature is $-53^{\circ} \mathrm{C}$. The numbers of data considered, over eight years, are 22596 for DC and 20814 for SP. 

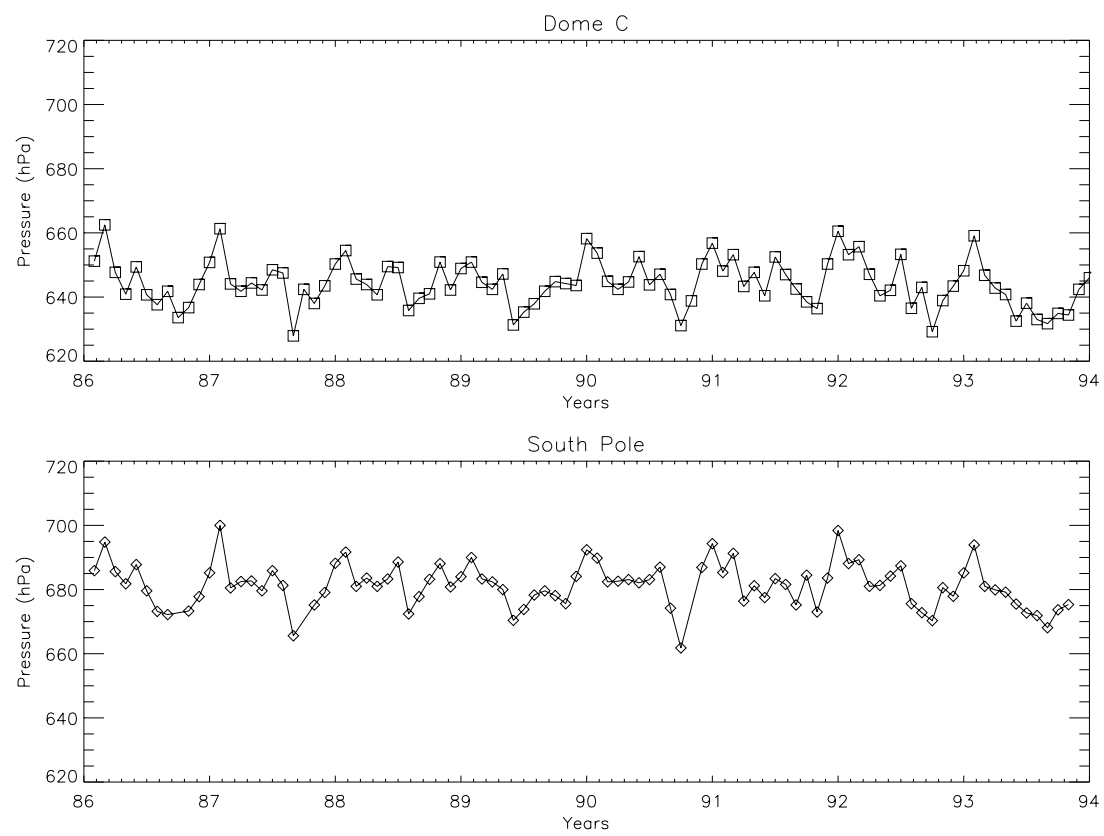

Figure 3-Average pressures for DC (upper panel) and SP (lower panel). The correlation between the two data sets is $92 \%$.

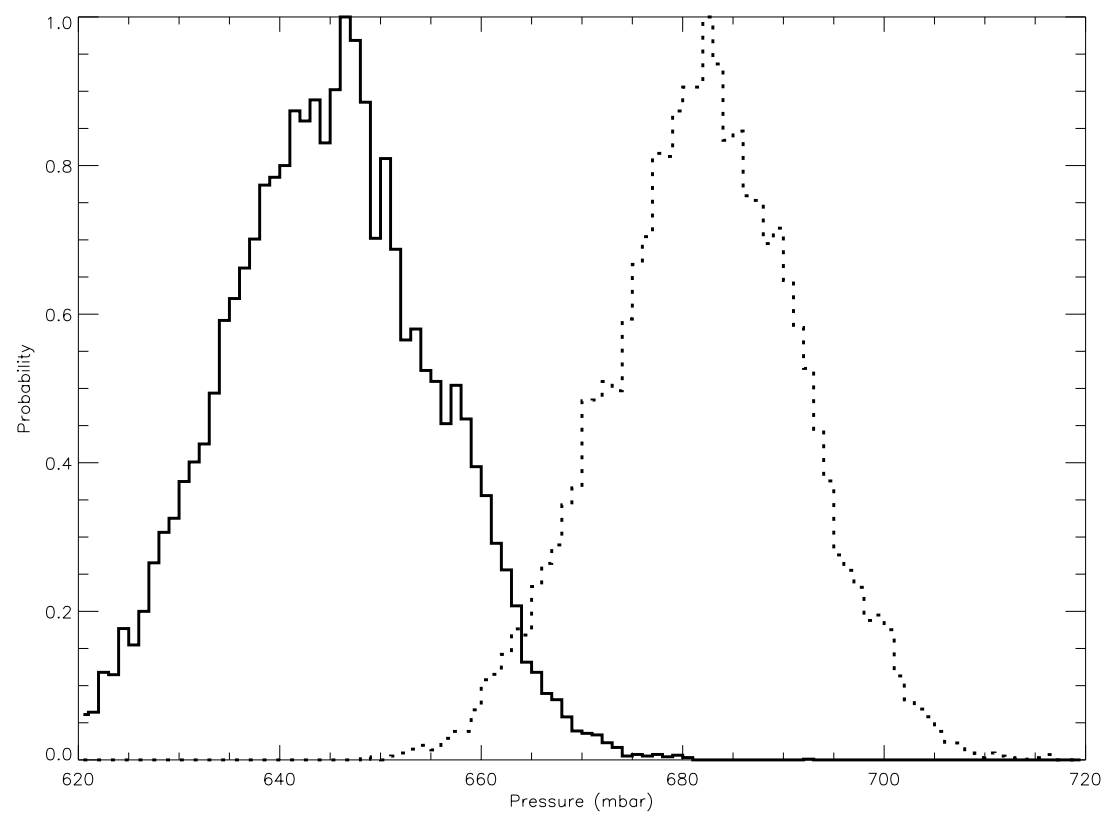

Figure 4-Pressure distributions for DC (continuous line) and SP (dotted line). Median values are $644 \mathrm{hPa}$ (22600 data) and $682 \mathrm{hPa}$ (20808 data) respectively. 

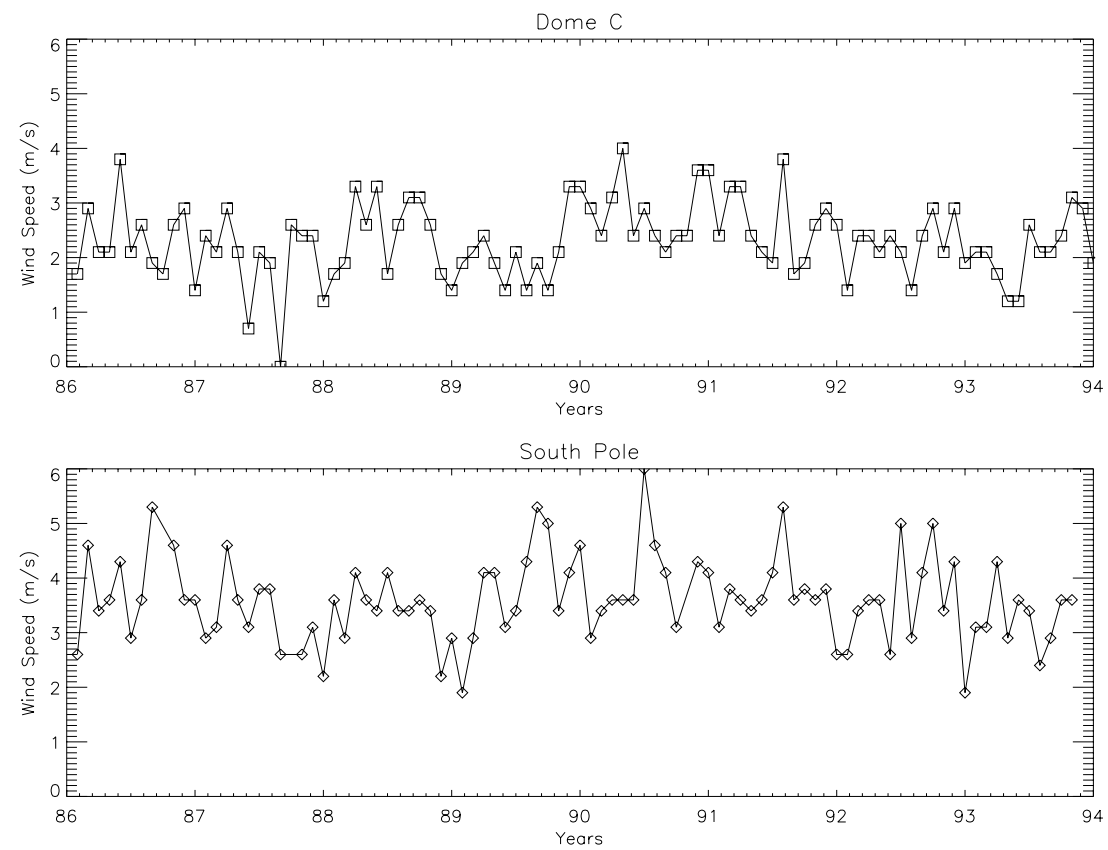

Figure 5-Average wind speeds for DC (upper panel) and SP (lower panel). The correlation between data sets is less than $30 \%$.

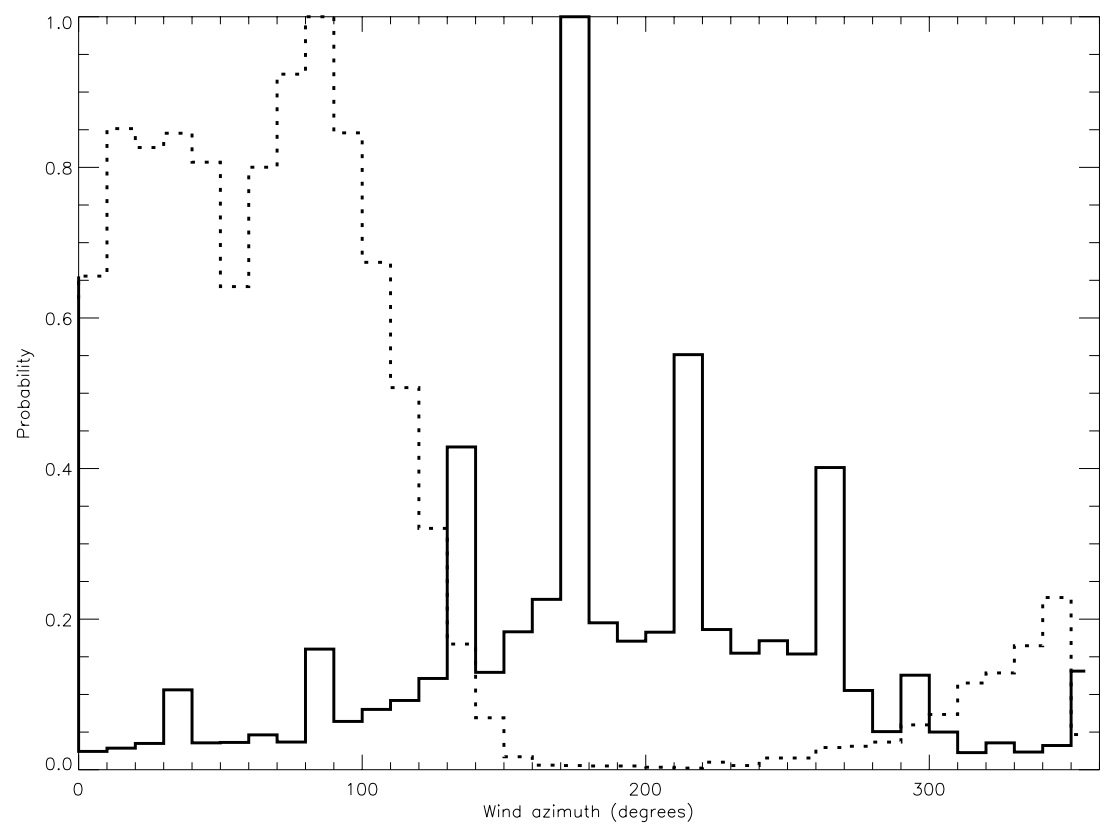

Figure 6-Wind azimuth distributions for DC (continuous line) and SP (dotted line). The distribution for DC (23034 points) is peaked around azimuth 180, while the SP data (20 784) are more uniformly distributed between azimuth 0 and azimuth 100 . 


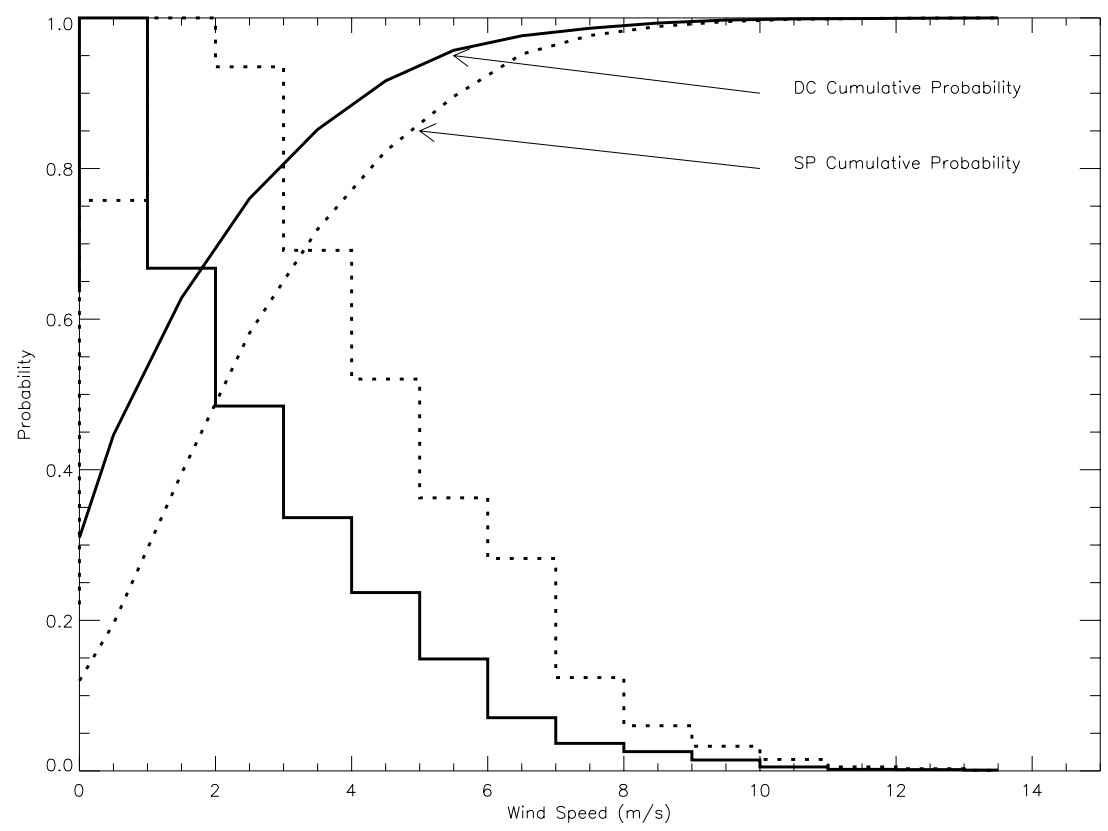

Figure 7-Histogram of data, with cumulative distribution of wind speed over-plotted, for DC (continuous line) and SP (dashed line). The numbers of data considered are 23038 and 20782 respectively. Distributions are not symmetric. The 50 th percentile $(0.5$ cumulative probability of measuring lower values) is $1 \mathrm{~m} \mathrm{~s}^{-1}$ for DC and $2 \mathrm{~m} \mathrm{~s}^{-1}$ for SP.

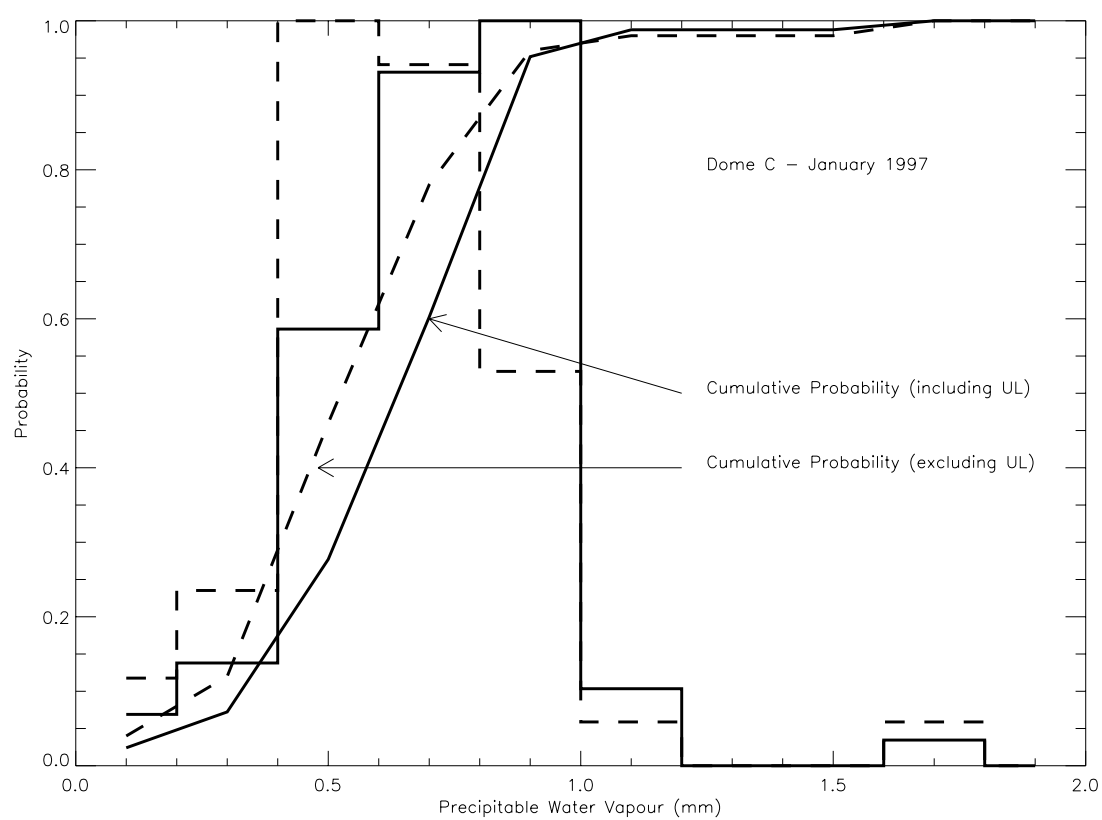

Figure 8-Precipitable Water Vapour data measured at DC during January 1997. The continuous line refers to the whole data set (83 measurements), including upper limits. The dashed-line histogram is calculated excluding upper limits (50 measurements). Cumulative probabilities are also plotted, with the same line styles. 
The PWV content, measured at DC during the 1996-97 Antarctic summer, is comparable to that at SP during summertime. The high Antarctic Plateau is shown to be the driest site compared to other observing sites. Further measurements at DC, with improved sensitivity and automatic operation of the instrument, are mandatory in order to explore conditions during wintertime also.

In conclusion, the overall exceptional quality of the high Antarctic Plateau at millimetre wavelengths, widely discussed in the literature (Dragovan et al. 1990; Chamberlin \& Bally 1994; Burton et al. 1994; Chamberlin 1995; Chamberlin, Lane \& Stark 1997; Lane 1998; Stark 1998), is confirmed by our work. DC conditions are at least comparable to, and probably better than, those at SP. However, further and more sophisticated site testing experiments are required to completely assess the DC observing quality.

\section{Acknowledgments}

Data were provided by the Automatic Weather Station Project, run by Dr Charles R. Stearns at the University of Wisconsin, Madison, which is funded by the National Science Foundation of the United States of America. Data are available by anonymous ftp at ice.ssec.wisc.edu. This work is partly supported by PNRA. We wish to thank G. Pizzichini, E. Pian and J. Stephen for their careful revision of the text.

\section{References}

Ade, P. A. R. 1984, Infr. Phys., 24, 403

Andreani, P., et al. 1990, Infr. Phys., 30, 479

Argentini, S. 1998, in Atti della Conferenza Nazionale sull'Antartide (Rome: PNRA)
Balm, S. P. 1996, PASA, 13, 14

Burton, M. G., et al. 1994, PASA, 11, 127

Burton, M. G. 1995, in Airborne Astronomy Symp. on the Galactic Ecosystem: From Gas to Stars to Dust, ASP Conf. Ser. 73, ed. M. R. Haas, J. A. Davidson \& E. F. Erickson (San Francisco: ASP), p. 559

Chamberlin, R. A. 1995, Int. J. Infr. Millim. Waves, 16, 907 Chamberlin, R. A., \& Bally, J. 1994, Appl. Opt., 33, 1095

Chamberlin, R. A., Lane, A. P. \& Stark, A. A. 1997, ApJ, 476, 428

Dall'Oglio, G. 1997, Communication at the Concordia Project Meeting, Siena (Italy), June 3-6

Dall'Oglio, G., et al. 1988, Infr. Phys., 28, 155

Dall'Oglio, G., et al. 1992, Exp. Astr., 2, 275

Dragovan, M., Stark, A. A., Pernic, R., \& Pomerantz, M. A. 1990, Appl. Opt., 29, 463

Gundersen, J. O., et al. 1995, ApJ, 443, L57

Holdaway, M. A., et al. 1996, MMA Memo 159

Keller, L. M., et al. 1997, Antarctic AWS Data for Calendar Year 1995, Space Science and Engineering Center, Univ. of Wisconsin, Madison WI, USA

Lane, A. P. 1998, in Astrophysics from Antarctica, ASP Conf. Ser. 141, ed. R. Landsberg \& G. Novak (San Francisco: ASP), p. 289

Ruhl, J. R., et al. 1995, ApJ, 451, L1

Smoot, G. F., et al. 1987, Radio Sci., 22, 521

Stark, A. 1998, in Astrophysics from Antarctica, ASP Conf. Ser. 141, ed. R. Landsberg \& G. Novak (San Francisco: ASP), p. 349

Storey, J. W. V., Ashley, M. C. B., \& Burton, M. G. 1996, PASA, 13, 35

Townes, C. H., \& Melnick, G. 1990, PASP, 102, 357

Valenziano, L. 1996, PhD thesis, Università di Perugia

Valenziano, L. 1997, TeSRE Rep. 192/97, available at http://tonno.tesre.bo.cnr.it/ valenzia/APACHE/ apache.htm

Valenziano, L., et al. 1997, Proc. PPEUC, available at http://www.mrao.cam.ac.uk/ppeuc/astronomy/papers/ valenziano/valenziano.html

Valenziano, L., et al. 1998, in Astrophysics from Antarctica, ASP Conf. Ser. 141, ed. R. Landsberg \& G. Novak (San Francisco: ASP), p. 81

Viper Home Page, http://cmbr.phys.cmu.edu/vip.html

Volz, F. 1974, Appl. Opt., 13, 1732 